\title{
ON SOME NEW SEQUENCE SPACES OF INVARIANT MEANS DEFINED BY ORLICZ FUNCTIONS
}

\author{
E. SAVAS* AND B. E. RHOADES
}

\begin{abstract}
The purpose of this paper is to introduce and study some sequence spaces which are defined by combining the concepts of a Orlicz function, invariant mean and lacunary convergence. We also examine some topological properties of these spaces and establish some elementary connections between lacunary $[w]_{\sigma}$-convergence and lacunary $[w] \sigma$-convergence with respect to an Orlicz functions which satisfy a $\Delta_{2}$-condition.
\end{abstract}

Mathematics subject classification (2000): Primary 46A45, 40H05; Secondary 40A05, 40D25. Key words and phrases: Lacunary sequence, invariant mean, Orlicz function.

\section{REFERENCES}

[1] S. BANaCh, Theorie des operations linearies, Warszawa, 1932.

[2] G. Das And S. Mishra, Lacunary distribution of sequences, Indian J. Pure Appl. Math. 20 (1) (1989), 64-74.

[3] G. DaS And J. K. SAhoo, On some sequence spaces, J. Math. Anal. Appl. 164 (1992), 381-398.

[4] A. R. Freedman, J. J. Sember AND M. RAPHAEL, Some Cesaro-type summability spaces, Proc. London Math. Soc. 37 (3) (1978), 508-520.

[5] P. K. Kamthan AND M. GuPTA, Sequence spaces and series, Marcel Dekker, New York, 1981.

[6] M. A. KRASNOSELSKII AND Y. B. RUTITSKY, Convex function and Orlicz spaces, Groningen, Netherlands, 1961.

[7] J. Lindenstrauss AND L. TZAFRIRI, On Orlicz sequence spaces, Israel J. Math. 10 (3) (1971), 379-390.

[8] G. G. LORENTZ, A contribution to the theory of divergent series, Acts Math. 80 (1948), 167-190.

[9] I. J. MADDOX, Spaces of strognly summable sequences, Quart. J. Math. Oxford 18 (2) (1967), 345-355. [10] $345-350$.

[11] MURSALEEN, On some new invariant matrix methods of summability, Quart. J. Math. Oxford 34 (1983), $77-86$.

[12] MursaleEN, Matrix transformation between some new sequence spaces, Houston J. Math. 9 (1983), 505-509.

[13] MursaleEn, A. K. GaUR AND T. A. ChISHTI, On some new sequence spaces of invariant means, Acta Math. Hungar. 75 (3) (1997), 209-214.

[14] S. D. Parashar and B. Chaudhary, Sequence spaces defined by Orlicz functions, Indian J. Pure appl. Math. 25 (1994), 419-428.

[15] W. H. RUCKLE, FK spaces in which the sequence of coordinate vectors is bounded, Can. J. Math. 25 (1973), 973-978.

[16] E. SAVAS, On lacunary strong $\sigma$-convergence, Indian J. Pure appl. Math. 21 (4) (1990), 359-365.

[17] E. SAVAS, On strongly $\sigma$-convergent sequences, Bull. Cal. Math. Soc. 81 (1989), 295-300.

[18] P. SCHAEFER, Infinite matrices and invariant means, Proc. Amer. Math. Soc. 36 (1972), 104-110. 